\title{
Implementation of a Pharmacist-Administered Influenza Vaccination Program in the Outpatient Setting at the Military Treatment Facility
}

Sean K. O'Brien, PharmD, MPH, BCPS'; Jennifer Bellisario, PharmD, BCPS ${ }^{2}$

${ }^{1}$ United States Army; ${ }^{2}$ Irwin Army Community Hospital, Fort Riley, Kansas

\begin{abstract}
The goal of the Army's influenza prevention program is to protect all Soldiers, healthcare professionals, and beneficiaries from influenza and its complications to maximize force readiness. Commanders have the daunting and challenging task of ensuring $100 \%$ of Soldiers and Military Treatment Facility (MTF) assigned employees are vaccinated against influenza by a specified timeframe. Outpatient Pharmacy Services at the MTF are in the perfect position to provide enhanced accessibility to the influenza vaccination to assist in meeting this requirement. Irwin Army Community Hospital at Fort Riley, Kansas, became the first MTF to offer a pharmacistadministered influenza vaccination program in the outpatient setting, as a walk-in service to all Soldiers, MTF employees, and beneficiaries, in 2016. Since then over 1,500 flu shots have been administered with no documented adverse events, and a potential cost-savings between $\$ 23,205$ to $\$ 61,880$. Here we describe the planning and implementation of such a program, which can easily be reproduced at any MTF outpatient pharmacy with minimal efforts and resources, enabling outpatient pharmacists to provide a convenient and accessible alternative for seasonal flu vaccination, with the ultimate goal of maximizing force readiness.
\end{abstract}

Keywords: Influenza vaccination; Military Treatment Facility; Outpatient pharmacists

\section{Introduction}

The flu, a contagious respiratory illness, is caused by influenza viruses that infect the throat, nose, and sometimes the lung. ${ }^{1}$ The flu places a substantial burden on the health of people in the United States each year. The Centers for Disease Control and Prevention (CDC) estimates that influenza results in over 40 million cases, over 750,000 hospitalizations, and up to 50,000 deaths annually. ${ }^{2}$ Additionally, flu seasons are unpredictable, and have the potential to impact the Department of Defense (DoD) force readiness and mission. ${ }^{3}$ Vaccination is the primary prevention method in reducing the probability of developing influenza and its complications. ${ }^{2}$

The All Army Activities (ALARACT) 099/2017, 2017-2018 Influenza Prevention Program-Surveillance and Vaccination, provides guidance to ensure that Soldiers and beneficiaries have access to protection against influenza. The intent of the influenza program is to protect all Army Active Component (AC), Army National Guard (ARNG), U.S. Army Reserve (USAR) personnel, mission-essential civilians, healthcare professionals, and Tricare beneficiaries from influenza and its complications. ${ }^{3}$ The key task is to immediately vaccinate this personnel with the flu vaccination, excluding those with contraindications to it. Commanders must ensure $100 \%$ of AC, ARNG, USAR personnel, and all Medical Treatment Facility (MTF) assigned employees are vaccinated by a specified timeframe. This can be a daunting and challenging mission to accomplish, thus, enhanced accessibility to the vaccination ensures this requirement is met. $^{3}$ Outpatient Pharmacy Services at the MTF are in the perfect position to provide this enhanced accessibility to positively impact force readiness and improve vaccination rates.

Corresponding author: Sean K. O'Brien, PharmD, MPH, BCPS United States Army; Email: seanobrienrx@gmail.com
As one of the most accessible healthcare professionals, outpatient pharmacists at the MTF can be instrumental in administering the influenza vaccination to Soldiers, MTF employees, and beneficiaries. ${ }^{4}$ Outpatient pharmacies at the MTF offer services on a walk-in, first-come first-served basis, without the need for an appointment. A flu vaccination program in this setting increases accessibility, while decreasing the burden of navigation throughout the healthcare system. In 1996, the American Pharmacists Association (APhA) established its Pharmacy-Based Immunization Delivery program. This CDC endorsed program teaches pharmacists the skills needed to become a primary provider for both vaccine education and administration. ${ }^{5}$ Although, all 50 states allow pharmacists to administer vaccinations, it is not common practice for outpatient pharmacists at the MTF to provide such services. In fact, Irwin Army Community Hospital (IACH) at Fort Riley, Kansas became the first MTF to provide the flu vaccination in the outpatient pharmacy, offering this service to all during pharmacy operating hours on a walk-in basis, in 2016 (Karla L. Gonzalez, Regional Health Command-Central, e-mail communication, October 2016).

The purpose of this article is to provide a comprehensive and detailed method, from the perspective of the first Army outpatient pharmacy to provide this service, for initiation of a pharmacist-administered influenza vaccination program in the outpatient pharmacy setting at the MTF, in an effort to enhance force readiness and vaccination rates.

Our Experience with Implementation of a PharmacistAdministered Influenza Vaccination Program in the Outpatient Setting at the MTF

Pharmacy Services at IACH followed the Commander's guidance to properly identify and educate Soldiers and beneficiaries to be vaccinated, track immunizations, and ensure 
appropriate medical evaluation of vaccinated personnel if they experience adverse reactions following vaccination. ${ }^{3}$ it is necessary and important to mention that Pharmacy Services first contacted and communicated with the Department of Public Health on Fort Riley to initiate the program. They were instrumental in directing the required training, assisting in hands-on competency training, and procuring the influenza vaccination.

\section{Planning}

\section{Step 1: Training Personnel}

First, outpatient pharmacists had to be trained to administer the influenza vaccination. A pharmacist was not required to have successfully completed APhA's Pharmacy-Based Immunization Delivery program, rather, they were required to complete the seasonal influenza training available by the Defense Health Agency (DHA). The trainings were available at https://health.mil/military-health-topics/health-

readiness/immunization-healthcare/education-and-training, and successful completion of the trainings titled, "Seasonal Influenza Vaccination Training," and "Seasonal Influenza Vaccine Cold Chain Management for Logistical Personnel," were required prior to administration. After the completion of these trainings, the pharmacist was required to conduct handson training, conducted initially by the Department of Public Health, and subsequently by previously trained pharmacy personnel, by completing the "Initial/Annual Competency Assessment Checklist: Injectable Influenza Vaccination." The training certificates of completion and competency were placed in the pharmacist's competency assessment file (CAF).

\section{Step 2: Preparing Forms}

In addition to the training needed to administer vaccinations, a memorandum for record (MFR) titled, "Vaccination Administration by Pharmacists at Irwin Army Community Hospital," was written by the Chief, Pharmacy Services, and provided to all outpatient pharmacists that completed the vaccination training. This MFR explained the liability coverage for the outpatient pharmacy would be covered by the DHA training, and not State Boards of Pharmacy. Furthermore, the Department of Public Health was instrumental in providing additional forms, including the Adult Screening and Immunization Worksheet, the Pediatric/Adolescent Screening and Immunization Worksheet, the 2017-2018 Seasonal Influenza Vaccine Documentation Card, and the Vaccine Information Sheet (VIS) for the Influenza Vaccine that is prepared by the CDC.

In an effort to minimize administration errors, and to easily differentiate the forms, pharmacy services reproduced the
Adult Screening and Immunization Worksheet on white paper, and the Pediatric/Adolescent Screening and Immunization Worksheet on green paper. The 2017-2018 Seasonal Influenza Vaccine Documentation Card, which are provided to the patient after administration of the flu vaccine, was reproduced on cardstock for durability. Finally, a copy the laminated VIS remained in the vaccination consultation room on display, and nonlaminated copies were available for the patient.

\section{Step 3: Obtaining a Standing Order and Collaboration with Privileged Clinical Pharmacist}

Another step in preparing to implement was obtaining a standing order from a provider to administer the influenza vaccination. ${ }^{6}$ A physician from the Department of Public Health provided this standing order. Additionally, a privileged clinical pharmacist in the patient-centered medical home (PCMH), who would be willing to co-sign the outpatient vaccination administration encounter in the Armed Forces Health Longitudinal Technology Application (AHLTA), was identified. Even though all of the privileged clinical pharmacists embedded in the $\mathrm{PCMH}$ were willing to assist, for simplicity and standardization of the process, one of the clinical pharmacists was designated to complete this task.

\section{Step 4: Establishing a Record-Keeping System}

AHLTA was used to document the patient's influenza administration by the outpatient pharmacist. Since outpatient pharmacists are not usually familiar with AHLTA documentation, clinical pharmacists were invaluable at providing this training. Additionally, it was crucial to ensure the vaccinating pharmacist updated the patient's Medical Protection System (MEDPROS), the system the Commanders use to ensure $100 \%$ of AC, ARNG, USAR personnel, and all MTF assigned employees are vaccinated. The Department of Public Health updated all of the AHLTA profiles for the vaccinating pharmacists to ensure successful transmission to MEDPROS once the AHLTA encounter was completed.

\section{Step 5: Ordering Supplies and Vaccines}

Table 1 depicts the necessary pharmacy supplies needed to administer the flu vaccine, which were ordered by pharmacy supply.

Additionally, Pharmacy Services created an emergency response kit (Table 2 ) in the event of an allergic reaction to the influenza vaccination. The lot numbers and expiration dates of these medications were tracked by inpatient pharmacy. 


\begin{tabular}{|l|l|}
\hline \multicolumn{2}{|c|}{ Table 1. Supplies and item description needed for influenza administration } \\
\hline Supply & Item Description \\
\hline Safety Needle & 25 Gauge $\times 1$ 1" hypodermic safety needle \\
\hline Alcohol Prep Pads & Individually wrapped 70\% isopropyl alcohol \\
\hline Gloves & Sensi-Guard powder-free exam gloves \\
\hline Gauze Sponges & $2^{\prime \prime} \times 2^{\prime \prime} 8$ ply gauze sponges \\
\hline Adhesive Bandages & All one size sheer strip adhesive bandage \\
\hline Tissue & Box of facial tissue \\
\hline Sharps Container & Wall mounted sharps container \\
\hline Hand Sanitizer & 8 ounce pump \\
\hline Miscellaneous & Trash can, pens, clip boards, plastic tackle box \\
\hline
\end{tabular}

\begin{tabular}{|l|l|}
\hline \multicolumn{2}{|c|}{ Table 2. Medications and quantity in emergency response kit } \\
\hline Medication & Quantity \\
\hline Diphenhydramine $25 \mathrm{mg}$ capsule & 4 \\
\hline Diphenhydramine $12.5 \mathrm{mg} / 5 \mathrm{~mL}$ unit-dosed cup & 4 \\
\hline Epinephrine (Adult) $0.3 \mathrm{mg}$ auto-injector & 2 \\
\hline Epinephrine (Child) $0.15 \mathrm{mg}$ auto-injector & 2 \\
\hline
\end{tabular}

Pharmacy Services ordered the influenza vaccination directly through the Department of Public Health. The initial quantity requested was 200 doses of the Flucelvax ${ }^{\circ}$, indicated for patients 4 years and older, and 100 doses of Fluarix ${ }^{\circ}$, indicated for patients 3 years and older. Both of these products are single dose, ready-to-use syringes. The vaccination was stored in a temperature-sensitive medical product (TSMP) monitored refrigerator in the outpatient pharmacy. When the vaccination supply diminished to approximately $50 \%$ of the original order, Pharmacy Services would request a replenishment from the Department of Public Health.

\section{Step 6: Setting up the Vaccination Area}

The next step in the preparation process involved setting up an appropriate site for the vaccination service in the outpatient pharmacy. Ideally, this area will provide privacy to protect patient confidentiality, and a comfortable environment. Figure 1 depicts the private consultation room adjacent to the waiting room in the outpatient pharmacy at IACH. Figure 2 depicts the necessary supplies and documents kept in the consultation room, including the emergency response kit. This private room provides an optimal vaccination site. If a consultation room is unavailable, a portable partition may be used for the vaccination area.

\section{Step 7: Getting the Word Out}

In order for this new service to be a success, it was necessary to partner with the Public Affairs Office (PAO). The information was disseminated by several different mechanisms, including IACH's social media webpage, a banner in the outpatient pharmacy, and flyers throughout the hospital, particularly in the PCMH. The hospital staff were aware of this new service, and when a patient presented to the $\mathrm{PCMH}$ or to the vaccination clinic, they informed the patient that outpatient pharmacy was another option for flu vaccine administration without the need for an appointment or wait.

\section{Implementation}

Implementation of this service was relatively simple once the initial set up and preparation was complete. When outpatient pharmacy received its influenza vaccination from the Department of Public Health, the service was launched. At this time, the option of a "Flu Shot" appeared on the queuing system kiosks in the outpatient pharmacy (Figure 3). This allowed the patient to select this option on a walk-in basis. $\mathrm{IACH}$ outpatient pharmacy uses a two-ticket-triage system, which involves the patient pulling a ticket, waiting in a lobby until their number is called to a pharmacy window, screening the patient's profile for the prescriptions to be filled, instructing the patient to sit back down in the lobby with the same ticket number that was called the first time, and calling the patient back to a pharmacy window once their prescriptions are verified to be dispensed.

The same process was used for patients that requested the "Flu Shot" option on the queuing system kiosk. When the patient was called to a pharmacy window for the first time, the pharmacy staff member provided the patient with the appropriate screening form (adult or pediatric) on a clipboard with a pen. To facilitate the flow through the outpatient pharmacy, the patient would take a seat while completing the form. Once complete, the patient would bring the form to any available pharmacy window. On average, IACH outpatient pharmacy has four pharmacists on duty during peak hours. At 
all times, one pharmacist was designated the vaccinating pharmacist, whose additional responsibility would be to administer the influenza vaccination. The vaccinating pharmacist screened the form for completeness and appropriateness for administration. The patient was then invited to the consultation room where they received an intramuscular shot of influenza vaccine. After vaccination, the pharmacist provided the patient with a completed seasonal influenza vaccine documentation card. As part of the postinjection monitoring program, patients were professionally instructed to remain in the pharmacy lobby area for 10 to 15 minutes after the injection. If the patient had any medications to fill in addition to the flu shot administration, this 10 to 15 minutes would be used to prepare and dispense them.

The vaccinating pharmacist maintained all screening worksheets in a folder located in the outpatient pharmacy. Approximately one hour before their shift ended, the pharmacist would document the influenza administration encounter into AHLTA, which would update the patient's MEDPROS. It is important to note that MEDPROS may take 48 to 72 hours to update, hence, providing the documentation card to the patient. Finally, the vaccinating pharmacist monitored the number of doses administered each day in a spreadsheet.

\section{Discussion}

During the 2016-2017 flu season, the first in which IACH offered this service, outpatient pharmacists vaccinated a total of 397 patients, delivering 238 (60\%) influenza vaccinations to MTF employees/beneficiaries, and 159 (40\%) to Soldiers. During the 2017-2018 flu season, outpatient pharmacists vaccinated 1,150 patients, delivering 761 (66\%) influenza vaccinations to MTF employees/beneficiaries, and $389(34 \%)$ to Soldiers. The average waiting time to receive an influenza vaccination was 12 minutes for the 2016-2017 flu season, and 9 minutes for the 2017-2018 flu season. During both flu seasons, no patient had an adverse reaction that required medical attention.

Furthermore, the cost savings for offering the influenza vaccination at the MTF is significant. Retail pharmacies are reimbursed an administration fee that can range from $\$ 15$ to $\$ 40$ per vaccination, depending on the state. ${ }^{7}$ Since inception of the $\mathrm{IACH}$ outpatient pharmacy influenza vaccination program, the cost savings has been between $\$ 23,205$ to $\$ 61,880$.

Our findings suggest that outpatient pharmacists at the MTF provide a highly convenient option for Soldiers and beneficiaries to receive the seasonal flu vaccination. As the first Army outpatient pharmacy to provide such a walk-in service, it is important to highlight some lessons learned so that other MTFs may successfully implement a similar program.

First, build your guiding coalition early. As previously mentioned, outpatient pharmacists at the MTF have not traditionally been trained to administer vaccinations, and this duty may seem daunting. However, civilian retail pharmacists have been providing this service for many years. One consistent message that was used to motivate the staff was that if $\mathrm{IACH}$ outpatient pharmacy did not offer the influenza vaccination, patients are likely to seek it at a civilian retail pharmacy by the same professional, and cost the taxpayer more money.

Second, expect the first year of a new program to be slower than subsequent years. IACH pharmacy discovered it takes times to get the word out, as shown by a three-fold in flu shot administration during the second year. As patients come to expect a service, the more popular it will be.

Finally, continuously think of ways to enhance this service. $\mathrm{IACH}$ is hoping to expand outpatient pharmacist vaccination services to include administration of other common vaccines, such as pneumococcal, hepatitis B, meningococcal, and others. Additionally, $\mathrm{IACH}$ is exploring the capability of allowing pharmacy technicians to administer certain vaccinations after proper training.

\section{Conclusions}

The Army's influenza program is to protect Soldiers, MTF employees, and beneficiaries from the complications of the flu by vaccination to ensure mission readiness. Pharmacists are highly trained and accessible healthcare professionals, and ideally positioned to provide the influenza vaccination in the outpatient setting at the MTF. Our findings suggest that outpatient pharmacists provide a convenient and accessible alternative for seasonal flu vaccination to assist Commanders in ensuring $100 \%$ of Soldiers and MTF employees are vaccinated. It is hoped that expanding outpatient pharmacist vaccination services to include administration of other common vaccines would further improve force readiness.

\section{Acknowledgements: None Conflicts of Interest: None Funding/Support: None}

The views expressed are solely those of the authors and do not reflect the official policy or position of the U.S. Army, Department of Defense, or the U.S. Government. 


\section{References}

1. Centers for Disease Control and Prevention. Key facts about influenza (flu). Available at: https://www.cdc.gov/flu/keyfacts.htm. Accessed November 12, 2017.

2. Centers for Disease Control and Prevention. Estimated influenza illnesses, medical visits, hospitalizations, and deaths averted by vaccination in the United States. Available at:

https://www.cdc.gov/flu/about/disease/2015-

16.htm. Accessed November 12, 2017.

3. All Armies Activities 099/2017: 2017-2018 Influenza

Prevention Program-Surveillance and Vaccination.

Fall Church, VA: Defense Health Agency; 2017.

Available at: https://health.mil/Military-Health-

Topics/Health-Readiness/Immunization-

Healthcare/Vaccine-Preventable-Diseases/Influenza-

Seasonal/Influenza-Resource-Center. Accessed

November 17, 2017.

4. Papastergiou J, Folkins C, Li W, Zervas J. Community pharmacist-administered influenza immunization improves patient access to vaccination. Canadian Pharmacists Journal. 2014;147(6):359-365. doi:10.1177/1715163514552557.

5. Terrie YC. Vaccinations: The expanding role of pharmacists. Pharmacy Times. 2010(1):1-8. Available at:

http://www.pharmacytimes.com/publications/issue/ 2010/january2010/ featurefocusvaccinations-0110. Accessed January 17, 2018.

6. Carter J, Slack M. A community pharmacy influenza vaccination service. In: Carter J, Slack $\mathrm{M}$, eds. Pharmacy in Public Health: Basics and Beyond. Bethesda, MD: American Society of Health-System Pharmacists; 2010:291-308.

7. Hartzell VA. Reimbursement for service of administering vaccines, from and independent pharmacy perspective. Pharmacy Times. 2017;8(2):S102-S105. Available at: http://www.pharmacytimes.com/publications/issue/ 2017/ immunizationsupplementjune2017/reimbursementfor-service-of-administering-vaccines-from-anindependent-pharmacy-perspective. Accessed January 26, 2018. 


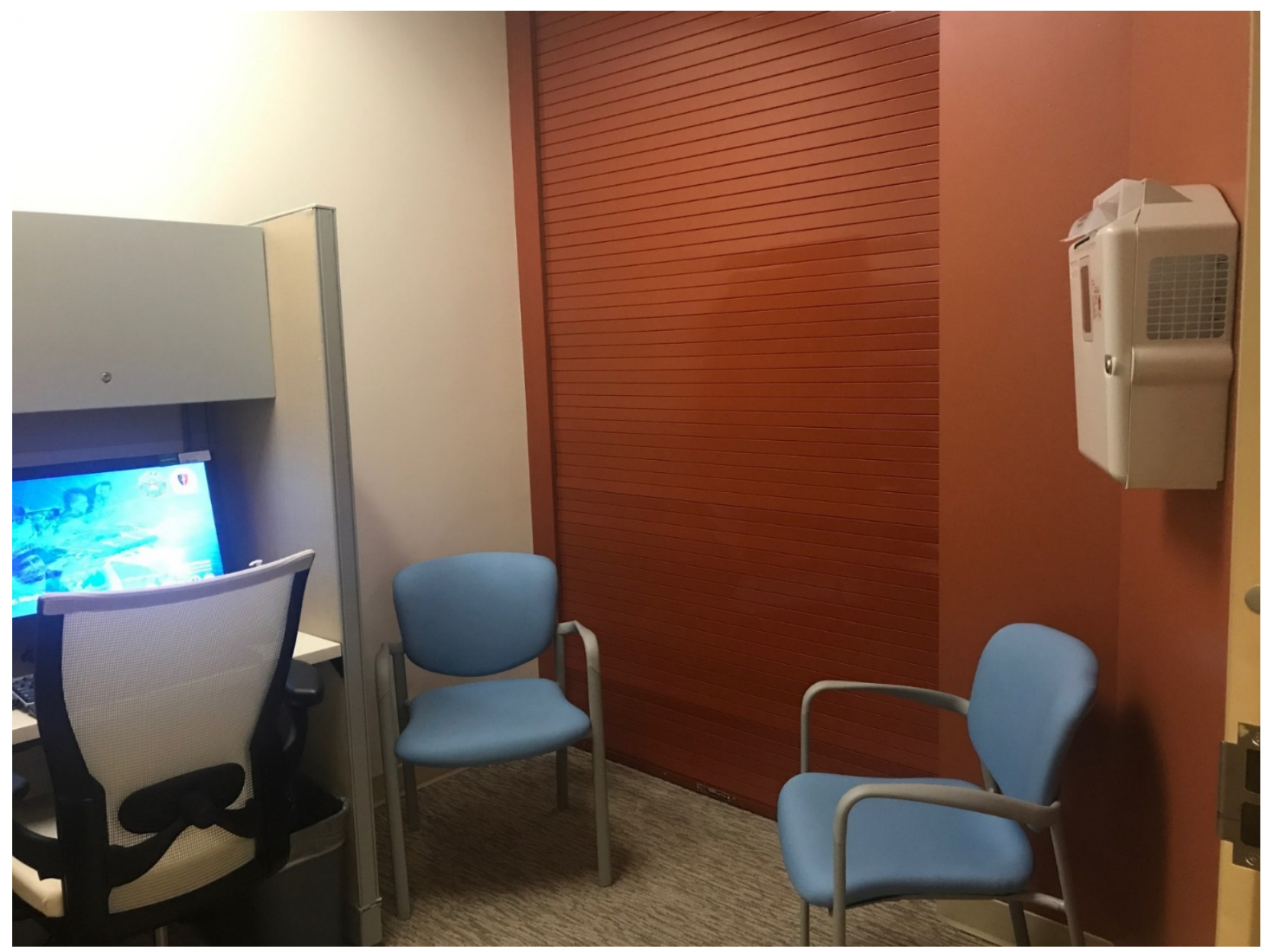

Figure 1. Private consultation room in the outpatient pharmacy for influenza vaccination administration. 


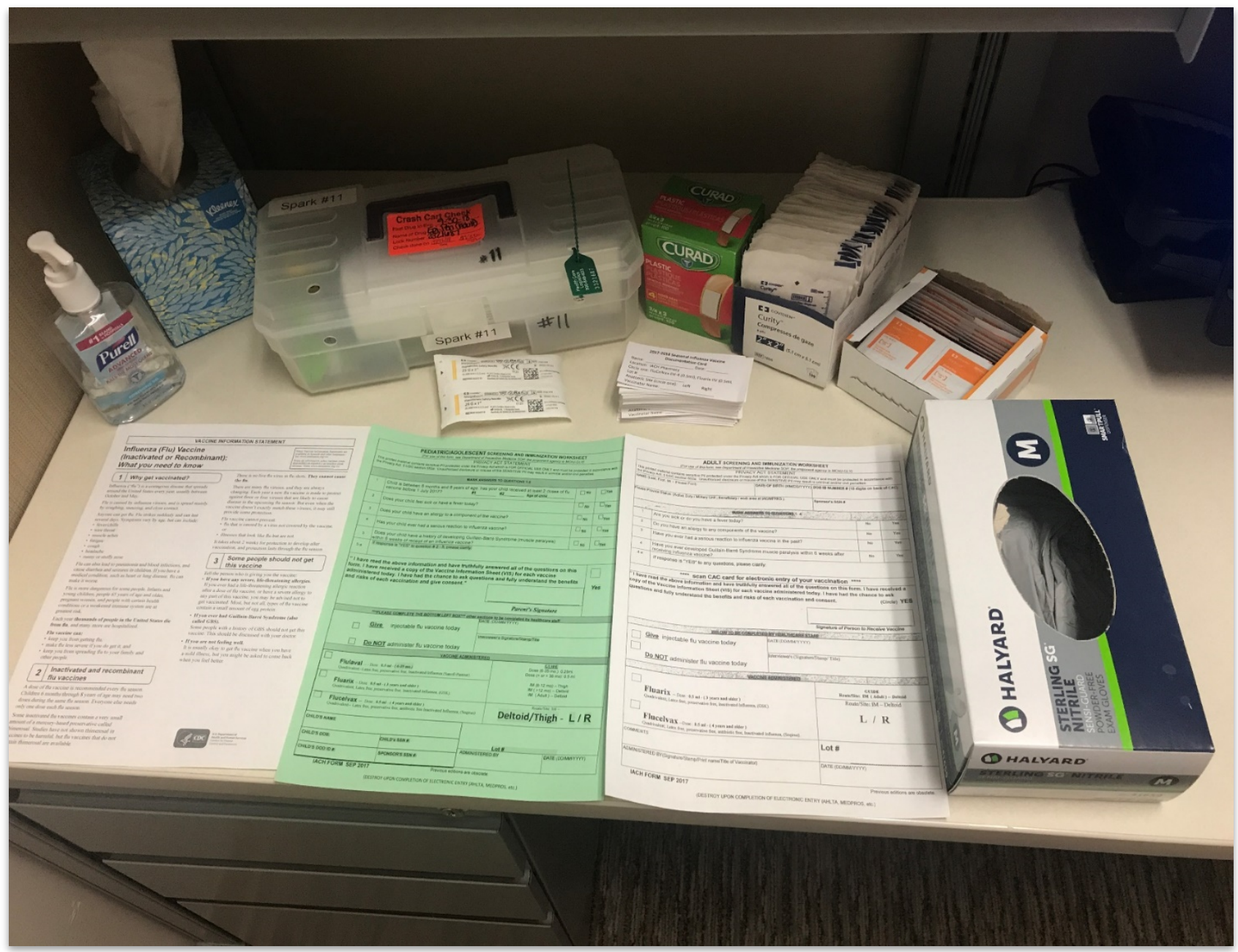

Figure 2. Necessary supplies to administer influenza vaccination in the outpatient pharmacy consultation room. 


\section{PLEASE SELECT AN OPTION BELOW}

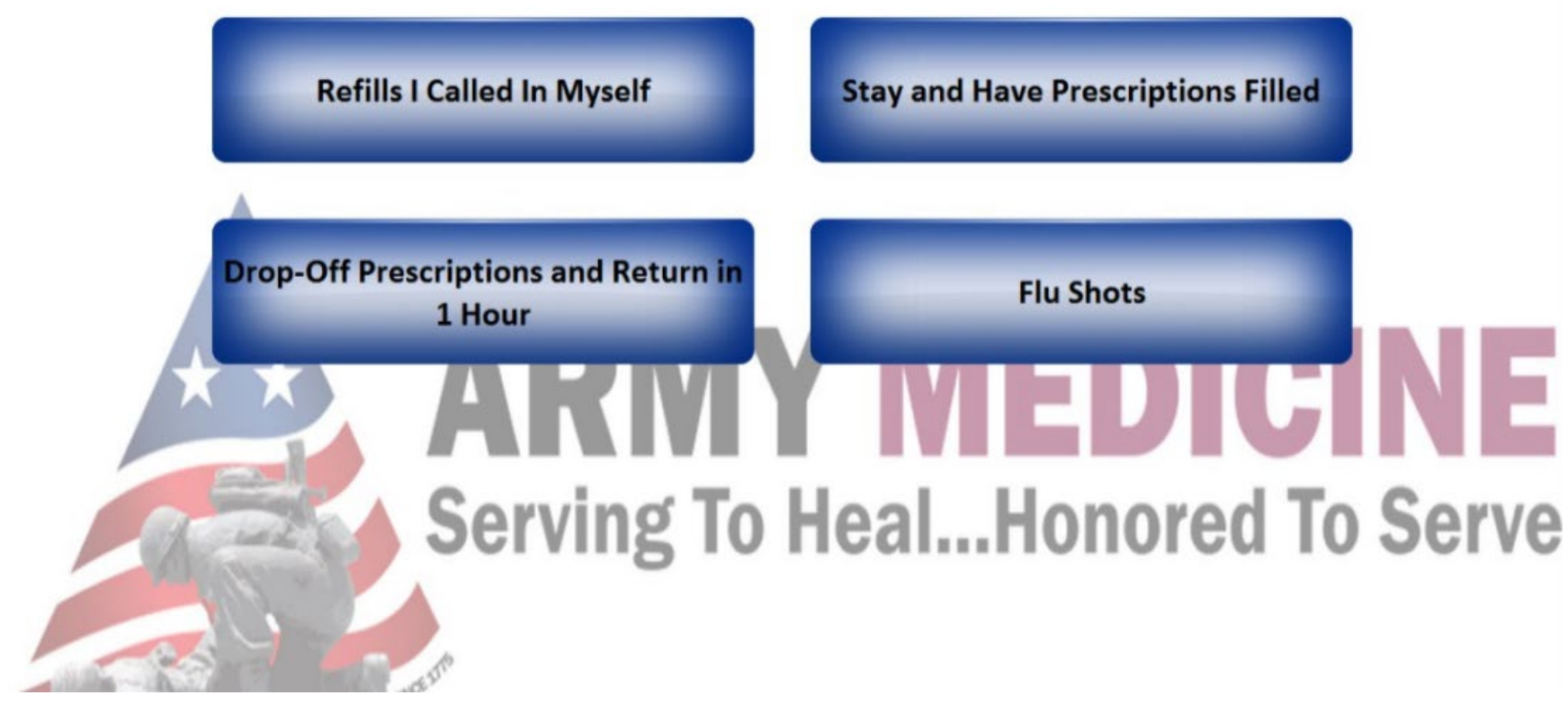

Figure 3. IACH outpatient pharmacy queuing system options displayed on kiosks, to include "Flu Shots" option. 